\title{
Simulation and image reconstruction of the combined Siemens PET/CT and PET/MRI systems
}

\author{
H. Kertesz ${ }^{1}$, A. Renner ${ }^{2}$, I. Rausch ${ }^{1}$, T. Beyer ${ }^{1}$, J. Cal-Gonzalez ${ }^{1}$ \\ ${ }^{1}$ QIMP group, Center for Medical Physics and Biomedical Engineering \\ ${ }^{2}$ Digital Image Processing Laboratory, Center for Medical Physics and Biomedical Engineering Medical \\ University of Vienna, Vienna, Austria \\ *e-mail:hunor.kertesz@meduniwien.ac.at
}

Key words: Monte Carlo simulation, performance evaluation, GATE simulation, NEMA protocol

Motivation and Aim: The objective of this work is to validate a Monte Carlo (MC) simulation model for two commercially-available, whole-body PET systems. The MC models will be used to evaluate the performance of different image reconstruction methodologies at low count rates.

Methods and Algorithms: GATE (GEANT4 Application for Tomographic Emission) was used as the MC toolkit for the modeling of the Siemens Biograph 64 TruePoint TrueView PET/CT (TPTV) and the Siemens Biograph PET/MR (mMR) systems. In both cases, we included detailed models of the detector electronics, system geometry and the physical processes involved in the data acquisition. The performance of both system models was validated following the NEMA (National Electrical Manufacturers Association) NU 2-2012 protocol. We compared the simulation results with the measured values for sensitivity, count rate (CR), and noise equivalent count rate (NECR). Moreover, three voxelized NEMA IQ phantom was simulated. The simulated data was reconstructed with the STIR framework using the standard OSEM algorithm.

Results: The calculated (reference value from measurements) sensitivity for the mMR was $13.8(15.0) \mathrm{kcps} / \mathrm{MBq}$ and $14.4(13.9) \mathrm{kcps} / \mathrm{MBq}$ at the center of the field-of-view (FOV) and at $10 \mathrm{~cm}$ radial offset, respectively. The NECR peak was $189 \mathrm{kcps} @ 23.8 \mathrm{kBq} / \mathrm{ml}$ (184 kcps@23.0 kBq/ml) and the scatter fraction at the NECR peak was 29.0 (37.9) \%. For the TPTV, the sensitivity was $8.0(8.1) \mathrm{kcps} / \mathrm{MBq}$ and $7.9(8.2) \mathrm{kcps} / \mathrm{MBq}$ at the centre of FOV and at $10 \mathrm{~cm}$ radial offset, respectively. The NECR peak was $151 \mathrm{kcps}$ @ $27 \mathrm{kBq} / \mathrm{ml}(161 \mathrm{kcps} @ 31 \mathrm{kBq} / \mathrm{ml})$ and the scatter fraction at the NECR peak was $24.8(32.5) \%$.

Conclusion: Both PET/CT and PET/MRI models showed a good agreement $(<10 \%)$ with the measured reference values. The application of these models for the evaluation of different image reconstruction algorithms in simulated numerical phantoms is work in progress.

Acknowledgements: The financial support of the Austrian FWF Project I3451- N32 is gratefully acknowledged. The computational results presented have been achieved using the Vienna Scientific Cluster (VSC). 